\title{
Inhaltsverzeichnis
}

\author{
Einleitung
}

Selte

$\S 1$ Die Aufgabe: Begriff und Eigenart des Handelsrechts - Das Schiffahrtsrecht — Plan der Darstellung . . . . . . . . . . . . 1 Anhang: Wirtschaftsrecht ................ . . . 8

\$ 2 Geschichte des Handelsrechts . . . . . . . . . . . . . . . . . . . 8

$\S 3$ Rechtsgrundlagen des deutschen Handelsrechts (Gesetze, Gewohnheitsrecht, Handelssitte, Verkehrssitte und Geschäftsbedingungen) . . . . 21

§ 4 Schrifttum zum deutschen Handelsrecht . . . . . . . . . . . . . . 29

$\S 5$ Quellen und Schrifttum des internationalen und ausländischen Handelsrechts ..................... 32

\section{Erster Abschnitt}

\section{Der Kaufmann und sein Handelsunternehmen}

\section{Erstes Kapitel: Die Kaufmannseigenschait}

$\S 6$ Allgemeines . . . . . . . . . . . . . . . . . . . . . . . . . . 39

$\S 7$ Der Mußkaufmann . . . . . . . . . . . . . . . . . . . . . . 40

$\S 8$ Weiteres zum Begriff des Mußkaufmanns . . . . . . . . . . . . . . . . 47

$\S 9$ Sollkaufleute . . . . . . . . . . . . . . . . . . . . . . 50

$\$ 10$ Land- und Forstwirte: Kannkaufleute . . . . . . . . . . . . . . . . . . 52

$\S 11$ Voll- und Minderkaufleute . . . . . . . . . . . . . . . . . . . . 55

Zweites Kapitel: Das Handelsregister und seine Ergänzung des Verkehrsschutzes - Der Seheinkaufmann

$\S 12$ Das Handelsregister und seine Ergänzungen des Verkehrsschutzes im allgemeinen . . . . . . . . . . . . . . . . 57

$\S 13$ Der Scheinkaufmann . . . . . . . . . . . . . . . . . . 66

Drittes Kapitel: Das Handelsgeschält (Handelsunternehmen im engeren Sinn)

$\S 14$ Begriff, rechtliche Natur, Rechtssätze. Das Ausland. . . . . . . . . 71

$\S 15$ Die Zweigniederlassung . . . . . . . . . . . . . . . . . . 81

$\S 16$ Das Handelsgeschäft als Gegenstand des Rechtsverkehrs . . . . . . 84

$\S 17$ Die Firma . . . . . . . . . . . . . . . . . . . . . . . . . . . 94

$\S 18$ Das Warenzeichen . . . . . . . . . . . . . . . . . 104

$\S 19$ Der unlautere Wettbewerb . . . . . . . . . . . . . . 112

$\S 20$ Die kaufmännische Buchführung . . . . . . . . . . . 119 
Viertes Kapitel: Die besondere Stellvertretung des Kaufmanns

$\$ 21$ Die Prokura. . . . . . . . . . . . . . . . . . . . . . 129

$\S 22$ Die Handlungsvollmacht . . . . . . . . . . . . . . . . . . . . . 134

Fünftes Kapitel: Unselbständige Hilfspersonen des Kaufmanns: Das kraufmännische Personal

$\S 23$ Allgemeines . . . . . . . . . . . . . . . . . . . . 139

$\S 24$ Die Handlungsgehilfen . . . . . . . . . . . . . . . . . . . . . . . 140

$\S 25$ Die Handlungslehrlinge . . . . . . . . . . . . . . . . . . . . . 147

Anhang: zu §§ 23-25: Der Handelsvolontär . . . . . . . . . . . 149

Sechstes Kapitel: Selbständige Hilfspersonen des Kaufmanns: Die Handelsvertreter und Handelsmakler

$\$ 26$ Die Handelsvertreter . . . . . . . . . . . . . . . . . . . . . . . 150

$\S 27$ Der Handelsmakler . . . . . . . . . . . . . . . . . . 160

Zweiter Abschnitt

\section{Die Gesellschaften des Handelsrechts}

Einleitung

$\S 28$ Begriff und Arten der „Gesellschaften“ . . . . . . . . . . . . . . 167

$\S 29$ Eigenart und Inhalt des Gesellschaftsrechts im allgemeinen . . . . . 170

$\S 30$ Die besonderen Gesellschaftsformen des Handelsrechts. . . . . . . . 175

Erstes Kapitel: Die Personalgesellschaiten im engeren Sinn des Handelsreehts

I. Die offene Handelsgesellschait (oHG)

$\S 31$ Begriff, rechtliche Natur, Geschichte, gesetzliche Regelung und

Eigenart . . . . . . . . . . . . . . . . 184

$\S 32$ Errichtung und Firma . . . . . . . . . . . . . . . . . . . 187

$\S 33$ Rechtsverhältnisse nach innen . . . . . . . . . . . . . . . . . . 194

$\S 34$ Rechtsverhältnisse zu Dritten . . . . . . . . . . . . . . . 206

$\S 35$ Beendigung, „Fortsetzung" . . . . . . . . . . . . . . . . . . . 212

$\S 36$ Ausscheiden und Eintritt von Gesellschaftern - Die zweigliedrige Gesellschaft . . . . . . . . . . . . . . . . 220

II. Die Kommanditgesellschait (KG)

$\S 37$ Begriff, Geschichte, wirtschaftliche Bedeutung, Rechtssätze . . . . . 225

III. Die stille Gesellschait (stG)

$\S 38$ Begriff, Geschichte, wirtschaftliche Bedeutung, Rechtssätze . . . . . 238

Z weites Kapitel: Die Kapitalgesellschaiten des Handelsrechts

I. Die Aktiengesellschait

§ 39 Begriff, Geschichte, die heutige gesetzliche Regelung in Deutschland, das Ausland, wirtschaftliche Bedeutung, Reformen. . . . . . . 247

$\S 40$ Die Grundlagen . . . . . . . . . . . . . . . . . 260 Anhang: Die Staatsaufsicht . . . . . . . . . . . 270 
$\S 41$ Gründung . . . . . . . . . . . . . . . . . . . 270

$\S 42$ Die Firma . . . . . . . . . . . . . . . . . . . . . . . 290

$\$ 43$ Organisation. . . . . . . . . . . . . . . . . . . 292

$\S 44$ Mitgliedschaft . . . . . . . . . . . . . . . . . . . . . . 322

$\S 45$ Rechtsverhältnisse zu Dritten . . . . . . . . . . . 342

$\S 46$ Jahresabschluß (Bilanz nebst Gewinn- und Verlustrechnung) und Geschäftsbericht . . . . . . . . . . . . . . . . 344

$\S 47$ Satzungsänderung, Kapitalerhöhung. Sonstige Maßnahme der Kapitalbeschaffung, Kapitalherabsetzung . . . . . . . . . . . 359

$\S 48$ Beendigung, „Fortsetzung", Sonderfälle (Verschmelzung von Kapitalgesellschaften, Verstaatlichung, Umwandlung). . . . . . . . . 374

\section{II.}

$\S 49$ Die Kommanditgesellschaft auf Aktien (KGaA). . . . . . . . . 396

III.

$\S 50$ Die Gesellschaft mit beschränkter Haftung (GmbH) . . . . . . . . 406

Drittes Kapitel: Soziale Wirtschaitsgenossenschaften

$\S 51$ Die eingetragene Genossenschaft . . . . . . . . . . . . . . . . 428

$\S 52$ Der Versicherungsverein auf Gegenseitigkeit (VVaG) . . . . . . 439

Viertes Kapitel:

$\S 53$ Die neuen freiwilligen wirtschaftlichen Zusammenschlüsse . . . . 444

Dritter Abschnitt

\section{Die Handelsgeschäfte}

\section{Erstes Kapitel: Allgemeine Lehren}

$\S 54$ Begriff und Arten der Handelsgeschäfte. Übersicht über die gesetzliche

Regelung . . . . . . . . . . . . . . . . 453

$\S 55$ Abschlu $B$ und Inhalt . . . . . . . . . . . . . . . . . . 456

$\S 56$ Der Eigentumserwerb .. . . . . . . . . . . . . . . 462

$\S 57$ Das Pfandrecht . . . . . . . . . . . . . . . . 465

$\S 58$ Das kaufmännische Zurückbehaltungsrecht . . . . . . . . . . . . . 466

$\mathrm{Z}$ weites Kapitel: Einzelne Handelsgeschäfte

$\S 59$ Einleitung. . . . . . . . . . . . . . . . . . . . 473

Erster Titel: Der Handelskauf

$\S 60$ Allgemeine Regeln . . . . . . . . . . . . . . . . . . 474

$\S 61$ Besondere Arten des Handelskaufs

Zweiter Titel: Die Bankgeschäfte

§ 62 Einleitung: . . . . . . . . . . . . . . . . . . . . . . 487

63 Kreditgeschäfte . . . . . . . . . . . . . . . . . . 491

\$ 64 Zahlungsgeschäfte . . . . . . . . . . . . . . . . . 500

$\$ 65$ Bankverwahrungsgeschäfte . . . . . . . . . . . . . . . 506

$\S 65$ a Geschäfte von Kapitalanlagegesellschaften in Deutschland . . . 511 
Dritter Titel: Börsengeschäftt

$\$ 66$ Börse und Börsenrecht. . . . . . . . . . . . . . . . 516

$\S 67$ Begriff, Arten, Behandlung der Börsengeschäfte. . . . . . . . . . 521

Vierter Titel: Das Kommissionsgeschält

$\S 68$ Begriff, rechtliche Natur, Ausland, Geschichte . . . . . . . . . . . 524

$\S 69$ Rechtssätze . . . . . . . . . . . . . . . . . . . . . 526

Fünfter Titel: Beförderungsgeschäfte (Transportgeschäfte)

$\S 70$ Begriff, Sonderrecht, Einteilung und Plan, Geschichte . . . . . . . 534

$\S 71$ Das Frachtgeschäft des HGB . . . . . . . . . . . . . . . . 537

$\S 72$ Das Eisenbahnfrachtgeschäft . . . . . . . . . . . . . . . 545

$\S 73$ Sonstige besondere Landfrachtgeschäfte . . . . . . . . . . . 553

\$ 74 Personenbeförderung zu Lande . . . . . . . . . . . . . . . . . 557

$\S 75$ Der Luftbeförderungsvertrag . . . . . . . . . . . . . . . . . . 558

$\S 76$ Das Speditionsgeschäft. . . . . . . . . . . . . . 560

Sechster Titel: Das Lagergeschäft

$\S 77$ Begriff, wirtschaftliche Bedeutung, Geschichte, Arten . . . . . . . . 569

$\S 78$ Das gewöhnliche Lagergeschäft und das Orderlagerscheingeschäft . . 571

Vierter Abschnitt

Das Schiffahrtsrecht

$\S 79$ Einleitung. . . . . . . . . . . . . . . . . . . . . . . . 577

Erstes Kapitel: Die Schiffe, der Reeder (Schifiseigner) und die Schiffsbesatzang

$\$ 80$ Die Schiffe . . . . . . . . . . . . . . . . . . . . . 583

$\S 81$ Reeder und Schiffseigner. . . . . . . . . . . . . . . 592

$\S 82$ Die Reederei (Partenreederei) . . . . . . . . . . . . . . . . . . 596

$\S 83$ Die Schiffsbesatzung des Seerechts .. . . . . . . . . . . . . 601

$\S 84$ Die Schiffsbesatzung des Binnenschiffahrtsrechtes . . . . . . 605

Zweites Kapitel: Beförderungsgeschäfte des Schiffahrtsrechtes

$\S 85$ Das Seefrachtgeschäft . . . . . . . . . . . . . . . . . 6 606

$\S 86$ Die Personenbeförderung zur See . . . . . . . . . . . . 620

$\S 87$ Das FluBfrachtgeschäft . . . . . . . . . . . . . . . . 620

Drittes Kapitel: Haverei und Hilfsleistung in Schiffahrtsnot

$\S 88$ Haverei und SchiffszusammenstoB . . . . . . . . . . . . . 622

$\S 89$ Hilfsleistung in Schiffahrtsnot. . . . . . . . . . . . . . . . . 626

Viertes Kapitel: Bodmerei, Schiffs- und Ladungsgläubiger

$\S 90$ Bodmerei . . . . . . . . . . . . . . . . . . . . 628

$\S 91$ Schiffs- und Ladungsgläubiger . . . . . . . . . . . . . . . . . . . . . . . . . . . 629

Sachverzeichnis. . . . . . . . . . . . . . 633 